\title{
DYNAMICS OF A STAGE-STRUCTURED POPULATION MODEL ON AN ISOLATED FINITE LATTICE*
}

\author{
Y. KYRYCHKO ${ }^{\dagger}$, S. A. GOURLEY ${ }^{\dagger}$, AND M. V. BARTUCCELLI ${ }^{\dagger}$
}

\begin{abstract}
In this paper we derive a stage-structured model for a single species on a finite onedimensional lattice. There is no migration into or from the lattice. The resulting system of equations, to be solved for the total adult population on each patch, is a system of delay equations involving the maturation delay for the species, and the delay term is nonlocal involving the population on all patches. We prove that the model has a positivity preserving property. The main theorems of the paper are comparison principles for the cases when the birth function is increasing and when the birth function is a nonmonotone function. Using these theorems we prove results on the global stability of a positive equilibrium.
\end{abstract}

Key words. nonlocal, stage structure, lattice, time delay, comparison

AMS subject classifications. 34K25, 34K12, 92D25

DOI. $10.1137 / \mathrm{S} 003614100444441 \mathrm{X}$

1. Background. There has recently been some interest in the study of stagestructured population models on lattices. The following model, which is of particular relevance to the present paper, was derived and studied by Weng, Huang, and Wu [8]:

$$
\begin{aligned}
\frac{d w_{j}(t)}{d t}= & \frac{\mu}{2 \pi} \sum_{k=-\infty}^{\infty} \mathcal{B}_{\alpha}(j-k) b\left(w_{k}(t-r)\right)+D_{m}\left[w_{j+1}(t)+w_{j-1}(t)-2 w_{j}(t)\right] \\
& -d_{m} w_{j}(t), \quad t>0, \quad j \in \mathbf{Z}
\end{aligned}
$$

where $j \in \mathbf{Z}:=\{0, \pm 1, \pm 2, \ldots\}$ are the integer nodes of an infinite one-dimensional lattice. The $r$-dependent parameters $\mu$ and $\alpha$ are given by

$$
\mu=\exp \left(-\int_{0}^{r} d(a) d a\right), \quad \alpha=\int_{0}^{r} D(a) d a
$$

with $d(a), D(a)$, and $r$ defined below, and the function $\mathcal{B}_{\alpha}(l)$ in (1.1), which we shall sometimes refer to as the kernel, is given by

$$
\mathcal{B}_{\alpha}(l)=2 e^{-2 \alpha} \int_{0}^{\pi} \cos (l \omega) e^{2 \alpha \cos \omega} d \omega .
$$

In their paper, Weng, Huang, and $\mathrm{Wu}[8]$ actually used the symbol $\beta$ for their kernel. However, it is important that their kernel should not be confused with the corresponding kernel for a finite lattice (which we are calling $\beta$ in this paper); thus we shall refer to the infinite lattice kernel of Weng, Huang, and $\mathrm{Wu}$ as $\mathcal{B}$.

In (1.1) the parameter $r$ measures the time from birth until reaching maturity and $w_{j}(t)$ denotes the total number of adults (i.e., the total number of age at least $r$ ) in the $j$ th patch. The function $b(\cdot)$, which always satisfies $b(0)=0$, is the birth function

\footnotetext{
*Received by the editors June 9, 2004; accepted for publication (in revised form) June 1, 2005; published electronically January 27, 2006.

http://www.siam.org/journals/sima/37-5/44441.html

${ }^{\dagger}$ Department of Mathematics and Statistics, University of Surrey, Guildford, Surrey GU2 7XH, U.K. (s.gourley@surrey.ac.uk).
} 
and the constants $D_{m}$ and $d_{m}$ are, respectively, the diffusion coefficient and death rate for the mature population.

In (1.2), the functions $D(a)$ and $d(a)$ are the diffusion coefficient and death rate for the immature population. For the immature population these rates can depend on age $a$, but for the mature population the diffusion coefficient and death rate must be independent of age and they are taken as $D_{m}$ and $d_{m}$.

Weng, Huang, and $\mathrm{Wu}[8]$ derived their model from the following von Foerster type of equation:

$$
\frac{\partial u_{j}}{\partial t}+\frac{\partial u_{j}}{\partial a}=D(a)\left[u_{j+1}(t, a)+u_{j-1}(t, a)-2 u_{j}(t, a)\right]-d(a) u_{j}(t, a)
$$

with $D(a)=D_{m}$ and $d(a)=d_{m}$ for $a \geq r$. Equation (1.4) incorporates a discrete representation of diffusion. Von Foerster equations for the case of continuous space have been considered also, in which case the Laplacian operator can be used to model Fickian diffusion (see [4]). In (1.4), $u_{j}(t, a)$ is the density of age $a$ at time $t$ in the $j$ th patch. Furthermore

$$
w_{j}(t)=\int_{r}^{\infty} u_{j}(t, a) d a
$$

In [8] the interest is mainly in the existence of travelling front solutions connecting two distinct equilibria. The highly nontrivial matter of the stability of these fronts is also investigated. Gourley and $\mathrm{Wu}$ [3] continued the study in [8] by providing conditions under which the population will go extinct, and conditions for the existence of periodic travelling waves.

The aim of the present paper is to derive and study an equation analogous to (1.1) for the case when the lattice is finite, with the nodes being given by $j=1,2, \ldots, N$. As we shall see, the model changes in two main respects. The first is that the discrete representation of diffusion will only be $w_{j+1}(t)+w_{j-1}(t)-2 w_{j}(t)$ at "interior" points of the lattice (i.e., the nodes $j=2,3, \ldots, N-1$ ) with a different expression for the nodes $j=1$ and $j=N$. We shall use the expression appropriate for an isolated lattice which individuals cannot escape from or enter into; this is the analogy of the homogeneous Neumann problem (i.e., no flux at boundaries) in the case of continuous space. The second and more complicated difference between the model of the present paper and (1.1) is that the term with the time delay assumes a rather different appearance. The function $\mathcal{B}_{\alpha}(l)$ given by (1.3) is completely inappropriate for the case of a finite lattice. Additionally, as we shall see, in the case of a finite lattice the time delay term no longer assumes a "convolution" structure (i.e., depending on the lattice index through the variables $j-k$ and $k$ with summation over $k$ ). This convolution formulation cannot allow for interactions with the endpoints of a finite lattice and therefore is strictly for infinite lattices only.

The derivation in [8] relies heavily on the fact that their lattice was infinite (their derivation utilizes a discrete Fourier transform technique). For a finite lattice a different model derivation is required and this will be the subject of the next section.

A model on a finite lattice similar to the one we propose in this paper was considered in Smith and Thieme [7]. Their model has $n$ patches each of which offers a different quality of life (the per capita reproduction rates, mortality rates, and maturation delays can vary from patch to patch). Additionally their model permits individuals to migrate from any one patch directly to any other. Their model assumes 
the form

$$
\left(\frac{\partial}{\partial t}+\frac{\partial}{\partial a}\right) u_{j}(t, a)=\sum_{k=1}^{n} \gamma_{j k} u_{k}(t, a)-\left(\sum_{k=1}^{n} \gamma_{k j}+\mu_{j}\right) u_{j}(t, a)
$$

with birth law

$$
u_{j}(t, 0)=g_{j}\left(\int_{\tau_{j}}^{\infty} u_{j}(t, a) d a\right)
$$

where each $g_{j}$ is a bounded function. In some respects this model is more general than the one we propose in this paper, particularly with regard to the migration terms ( $\gamma_{j k}$ is the per capita migration rate from node $k$ to node $j$ ). Their key assumption on the migration terms is that the matrix $\left(\gamma_{j k}\right)$ be irreducible. Our model in this paper considers migration only on a nearest neighbor basis, but we study our model in somewhat more detail.

Our approach is to start with an age-structured model (the "original problem") given by system (2.1) below, and derive from it a delay differential equation system (the "reduced problem") for $w_{j}(t)$, the total mature population at the $j$ th patch. As we shall discover, the reduced system (system (2.10) below) is valid only after a transient period of length $r$. For $0<t<r$ the variable $w_{j}(t)$ is governed by some different nonautonomous equations that involve the initial data $\mathbf{u}_{0}(a)$ for the original problem (2.1). We shall not be concerned in this paper with these other nonautonomous equations but will effectively neglect the transient phase and study the reduced problem independently (i.e., we study system (2.10) below for $t>0$, subject to (2.11)). However, there are delicate issues regarding initial data and positivity which will be discussed later in this paper and which are treated in more detail in Bocharov and Hadeler [2] for problems without diffusion. For example, only certain initial data for the reduced problem with delay are related to the original problem. Also, while positive solutions of the original problem lead to positive solutions of the reduced problem, the cone of positive solutions of the reduced problem is larger in general, since we study the reduced problem for arbitrary nonnegative initial data, and not just those initial data that are related to the original problem. These issues will be discussed further as they arise.

2. Finite lattice: Model derivation. Let $u_{j}(t, a)$ denote the density of the population of the species at the $j$ th patch at time $t \geq 0$ and age $a \geq 0$. Let $D(a)$ and $d(a)$ denote the diffusion and death rates of the population at age $a$. Assume that the patches are located at the integer nodes $j=1,2, \ldots, N$ of a one-dimensional lattice and that spatial diffusion occurs only at the nearest neighborhood and is proportional to the difference of the densities of the population at adjacent patches. These assumptions lead to the model

$$
\left(\frac{\partial}{\partial t}+\frac{\partial}{\partial a}\right) \mathbf{u}(t, a)=D(a) A \mathbf{u}(t, a)-d(a) \mathbf{u}(t, a)
$$

for $t>0$, where

$$
\mathbf{u}(t, a)=\left(u_{1}(t, a), \ldots, u_{N}(t, a)\right)^{T}
$$


and

$$
A=\left(\begin{array}{ccccccc}
-1 & 1 & 0 & \cdots & 0 & 0 & 0 \\
1 & -2 & 1 & \cdots & 0 & 0 & 0 \\
\vdots & \vdots & \vdots & & \vdots & \vdots & \vdots \\
0 & 0 & 0 & \cdots & 1 & -2 & 1 \\
0 & 0 & 0 & \cdots & 0 & 1 & -1
\end{array}\right)
$$

subject to

$$
u_{j}(t, 0)=b\left(w_{j}(t)\right)
$$

where $w_{j}(t)$ is the total mature population at the $j$ th patch, given by

$$
w_{j}(t)=\int_{r}^{\infty} u_{j}(t, a) d a
$$

and $b(\cdot)$ is the birth function, which satisfies $b(0)=0$. Furthermore, it is natural to assume that

$$
u_{j}(t, \infty)=0, \quad t \geq 0, \quad j=1, \ldots, N
$$

The initial data for (2.1) has the form

$$
\mathbf{u}(0, a)=\mathbf{u}_{0}(a), \quad 0 \leq a<\infty
$$

with $\mathbf{u}_{0}(a)$ prescribed.

Note that, at the node $j=1$, the diffusion term is $D(a)\left(u_{2}-u_{1}\right)$ with a similar expression for the other "end" node $j=N$. In this way the model (2.1) has been set up so as to be the discrete analogue of what is commonly called the homogeneous Neumann problem in the continuous case, in which no-flux boundary conditions are applied. For the heat equation $u_{t}=\Delta u$ on a finite domain $\Omega$ with homogeneous Neumann boundary conditions $\partial u / \partial n=0$ on $\partial \Omega$, it is well known that $\int_{\Omega} u(t, x) d x$ is constant. An analogous result holds for (2.1) in the case when there are no births or deaths (i.e., $b(\cdot)=d(\cdot)=0)$. Indeed, in this case,

$$
\begin{gathered}
\quad \frac{d}{d t} \underbrace{\sum_{j=1}^{N} \int_{0}^{\infty} u_{j}(t, a) d a}_{\text {total population }}=\sum_{j=1}^{N} \int_{0}^{\infty} \frac{\partial u_{j}(t, a)}{\partial t} d a=-\sum_{j=1}^{N} \int_{0}^{\infty} \frac{\partial u_{j}(t, a)}{\partial a} d a \\
+\sum_{j=2}^{N-1} \int_{0}^{\infty} D(a)\left(u_{j-1}(t, a)-2 u_{j}(t, a)+u_{j+1}(t, a)\right) d a \\
+\int_{0}^{\infty} D(a)\left(-u_{1}(t, a)+u_{2}(t, a)\right) d a+\int_{0}^{\infty} D(a)\left(u_{N-1}(t, a)-u_{N}(t, a)\right) d a \\
=-\sum_{j=1}^{N}(\underbrace{u_{j}(t, \infty)}_{=0}-\underbrace{b\left(w_{j}(t)\right)}_{=0 \text { if no births }})=0 .
\end{gathered}
$$

Our intention is to derive from (2.1), (2.3) a system of equations satisfied by the total matured population $w_{j}(t), j=1,2, \ldots, N$. Before doing so, let us introduce the 
function $\beta(t, k, j)$ defined by

$$
\beta(t, k, j)=\frac{1}{N}+\frac{2}{N} \sum_{l=1}^{N} e^{-4 \sin ^{2}\left(\frac{l \pi}{2 N}\right) t} \cos \left[(2 j-1) \frac{l \pi}{2 N}\right] \cos \left[(2 k-1) \frac{l \pi}{2 N}\right] .
$$

We will prove the following result which is useful for later calculations.

Proposition 2.1. The function $\beta(t, k, j)$ defined by $(2.6)$ has the following properties:

(i) it satisfies

$$
\frac{d}{d t}\left(\begin{array}{c}
\beta(t, k, 1) \\
\vdots \\
\beta(t, k, N)
\end{array}\right)=\left(\begin{array}{ccccccc}
-1 & 1 & 0 & \cdots & 0 & 0 & 0 \\
1 & -2 & 1 & \cdots & 0 & 0 & 0 \\
\vdots & \vdots & \vdots & & \vdots & \vdots & \vdots \\
0 & 0 & 0 & \cdots & 1 & -2 & 1 \\
0 & 0 & 0 & \cdots & 0 & 1 & -1
\end{array}\right)\left(\begin{array}{c}
\beta(t, k, 1) \\
\vdots \\
\beta(t, k, N)
\end{array}\right)
$$

(ii) $\beta(0, k, j)$ is the Kronecker delta:

$$
\beta(0, k, j)= \begin{cases}1 & \text { if } j=k \\ 0 & \text { if } j \neq k\end{cases}
$$

(iii) $\sum_{k=1}^{N} \beta(t, k, j)=1$ for each $j=1,2, \ldots, N$ and all $t \geq 0$;

(iv) $\beta(t, k, j)>0$ for all $t>0$ and all $1 \leq k, j \leq N$.

Proof. Property (i) is straightforward. Property (ii) is fairly easily seen. Indeed,

$$
\begin{aligned}
\beta(0, k, k) & =\frac{1}{N}+\frac{2}{N} \sum_{l=1}^{N} \cos ^{2}(2 k-1) \frac{l \pi}{2 N}=\frac{1}{N}+\frac{1}{N} \sum_{l=1}^{N}\left(1+\cos (2 k-1) \frac{l \pi}{N}\right) \\
& =\frac{1}{N}+1+\frac{1}{N} \operatorname{Re}\left(\sum_{l=1}^{N} e^{i(2 k-1) \frac{l \pi}{N}}\right) \\
& =\frac{1}{N}+1+\frac{2}{N} \operatorname{Re}\left(\frac{e^{i(2 k-1) \frac{\pi}{N}}}{1-e^{i(2 k-1) \frac{\pi}{N}}}\right) \\
& =1
\end{aligned}
$$

after some algebra. Similarly, $\beta(0, k, j)=0$ for $j \neq k$.

To show statement (iii) it is clearly sufficient to prove that

$$
\sum_{k=1}^{N} \cos (2 k-1) \frac{l \pi}{2 N}=0
$$

and this is easily shown.

Finally we prove (iv), that $\beta(t, k, j)>0$ for all $t>0$. We have already noted that the function $\beta(t, k, j)$ defined by (2.6) satisfies the system of differential equations (2.7) with the initial condition (2.8). Here, $j$ is thought of as the spatial coordinate and $k \in\{1,2, \ldots, N\}$ as fixed. Certain theorems in the theory of matrices (Berman and Plemmons [1]) are useful here. The matrix $\beta(t)=[\beta(t, k, j)]_{N \times N}$ is the solution of the linear system $\dot{\beta}=A \beta$, where $A$ is the matrix defined by $(2.2)$, subject to $\beta(0)=I$. Thus

$$
\beta(t)=e^{A t}=e^{-3 t} e^{(3 I+A) t}=e^{-3 t} \sum_{i=0}^{\infty} \frac{t^{i}(3 I+A)^{i}}{i !} .
$$


It is easily checked that $3 I+A$ is a positive and irreducible matrix. Therefore, it follows [1] that $(3 I+A)^{i}$ is a strictly positive matrix for all $i \geq N+1$. Therefore, for any $t>0$ the infinite sum above furnishes a matrix all of whose elements are strictly positive. Therefore, $\beta(t, k, j)>0$ for all $t>0$. The proof of Proposition 2.1 is complete.

We will now prove the following theorem.

THEOREM 2.2. Assume that the diffusion and death rates of the mature population are age-independent, i.e.,

$$
D(a)=D_{m}, \quad d(a)=d_{m} \quad \text { for } a \in[r, \infty),
$$

where $D_{m}>0$ and $d_{m}>0$ are constants. Let the function $\beta(t, k, j)$ be defined by (2.6) and let

$$
\mu=e^{-\int_{0}^{r} d(z) d z}, \quad \alpha=\int_{0}^{r} D(z) d z .
$$

Then for $t \geq r$ the total matured population $w_{j}(t)$ defined by (2.4) satisfies

$$
\begin{gathered}
\frac{d}{d t}\left(\begin{array}{l}
w_{1}(t) \\
w_{2}(t) \\
\vdots \\
w_{N-1}(t) \\
w_{N}(t)
\end{array}\right)=\mu \sum_{k=1}^{N} b\left(w_{k}(t-r)\right)\left(\begin{array}{l}
\beta(\alpha, k, 1) \\
\beta(\alpha, k, 2) \\
\vdots \\
\beta(\alpha, k, N-1) \\
\beta(\alpha, k, N)
\end{array}\right) \\
(2.10)+D_{m}\left(\begin{array}{ccccccc}
-1 & 1 & 0 & \cdots & 0 & 0 & 0 \\
1 & -2 & 1 & \cdots & 0 & 0 & 0 \\
\vdots & \vdots & \vdots & & \vdots & \vdots & \vdots \\
0 & 0 & 0 & \cdots & 1 & -2 & 1 \\
0 & 0 & 0 & \cdots & 0 & 1 & -1
\end{array}\right)\left(\begin{array}{l}
w_{1}(t) \\
w_{2}(t) \\
\vdots \\
w_{N-1}(t) \\
w_{N}(t)
\end{array}\right)-d_{m}\left(\begin{array}{l}
w_{1}(t) \\
w_{2}(t) \\
\vdots \\
w_{N-1}(t) \\
w_{N}(t)
\end{array}\right) .
\end{gathered}
$$

Remark 1. For $0<t<r$ the variable $w_{j}(t)$ does not obey system (2.10) but is governed instead by some different (nonautonomous) equations that still contain information about the initial data of the system (2.1). This issue is discussed in detail in Bocharov and Hadeler [2] for systems without diffusion. However, in this paper we shall concentrate on the problem consisting of system (2.10) for $t>0$, subject to the initial conditions

$$
w_{j}(s)=w_{j}^{0}(s) \geq 0, \quad j=1,2, \ldots, N, \quad s \in[-r, 0]
$$

with $w_{j}^{0}(s)$ prescribed.

Remark 2. Related to the above point, is the issue of initial data. If we solve (2.10) for $t>0$ an important question arises: does an arbitrary nonnegative initial function $w_{j}^{0}(s), s \in[-r, 0]$, necessarily result, in any sense, from an initial datum $\mathbf{u}_{0}(a)$ for system (2.1)? The answer is, not necessarily. Given an initial datum $\mathbf{u}_{0}(a)$ for system (2.1), one should first evolve the variable $w_{j}(t)$ until time $r$ according to the nonautonomous equations which govern $w_{j}(t)$ for $0<t<r$, and then evolve $w_{j}(t)$ for $t>r$ according to (2.10) with initial time $r$, using as initial data the values of $w_{j}(t), t \in[0, r]$, found by solving the nonautonomous equations. Those functions $w_{j}(t), t \in[0, r]$, which arise in this way are, once translated $r$ units back in time, those 
functions which are admissible in (2.11) as initial data for system (2.10) with initial time 0. For a class of systems without diffusion, Bocharov and Hadeler [2] characterize completely those initial data for their delay equation starting at time $r$, which result from a positive initial datum of their analogy to our system (2.1). In this paper we shall not develop this issue further but will consider (2.10) for $t>0$ subject only to (2.11) (i.e., as though the system had not been derived from a structured population model).

Proof of Theorem 2.2. Letting

$$
\mathbf{w}(t)=\left(w_{1}(t), \ldots, w_{N}(t)\right)^{T},
$$

we have

$$
\frac{d \mathbf{w}(t)}{d t}=\int_{r}^{\infty} \frac{\partial}{\partial t} \mathbf{u}(t, a) d a=\int_{r}^{\infty}\left[-\frac{\partial}{\partial a} \mathbf{u}(t, a)+D(a) A \mathbf{u}(t, a)-d(a) \mathbf{u}(t, a)\right] d a .
$$

We obtain from (2.1) and (2.12) that

$$
\frac{d \mathbf{w}(t)}{d t}=\mathbf{u}(t, r)+D_{m} A \mathbf{w}(t)-d_{m} \mathbf{w}(t) \quad \text { for } t>0 .
$$

In order to have a complete system for $w_{j}(t)$ we need to calculate $u_{j}(t, r), j=$ $1,2, \ldots, N$. For fixed $s \geq 0$ let $\mathbf{V}^{s}(t)=\left(V_{1}^{s}(t), \ldots, V_{N}^{s}(t)\right)^{T}$, where

$$
V_{j}^{s}(t)=u_{j}(t, t-s) \quad \text { for } s \leq t \leq s+r .
$$

Since only the mature population can reproduce, we have

$$
V_{j}^{s}(s)=u_{j}(s, 0)=b\left(w_{j}(s)\right)
$$

where $b: \mathbb{R}_{+} \rightarrow \mathbb{R}_{+}$is the birth function. From (2.1), we have

$$
\frac{d}{d t} \mathbf{V}^{s}(t)=\left.\left(\frac{\partial}{\partial t}+\frac{\partial}{\partial a}\right) \mathbf{u}(t, a)\right|_{a=t-s}=D(t-s) A \mathbf{V}^{s}(t)-d(t-s) \mathbf{V}^{s}(t) .
$$

We want to solve (2.16) subject to (2.15). First, let

$$
V_{j}^{s}(t)=\tilde{V}_{j}^{s}(t) e^{-\int_{0}^{t-s} d(z) d z}
$$

and $\tilde{\mathbf{V}}^{s}(t)=\left(\tilde{V}_{1}^{s}(t), \ldots, \tilde{V}_{N}^{s}(t)\right)^{T}$; then system (2.16) becomes

$$
\frac{d}{d t} \tilde{\mathbf{V}}^{s}(t)=D(t-s) A \tilde{\mathbf{V}}^{s}(t) .
$$

Making a further transformation of time $t$ as

$$
\tilde{t}=\int_{0}^{t-s} D(z) d z
$$

so that $d \tilde{t} / d t=D(t-s)$, we obtain

$$
\frac{d}{d \tilde{t}} \tilde{\mathbf{V}}^{s}(\tilde{t})=A \tilde{\mathbf{V}}^{s}(\tilde{t})
$$


which has to be solved subject to the initial condition (2.15) in the form

$$
\tilde{V}_{j}^{s}(0)=b\left(w_{j}(s)\right), \quad j=1,2, \ldots, N .
$$

We look for a solution of (2.17) in the form

$$
\tilde{V}_{j}^{s}(\tilde{t})=\sum_{k=1}^{N} c_{k} \beta(\tilde{t}, k, j),
$$

where the function $\beta(t, k, j)$ is defined in (2.6) and $c_{k}$ are unknown constants to be found using the initial condition (2.18). That is, with $\tilde{t}=0$ one gets

$$
\tilde{V}_{j}^{s}(0)=\sum_{k=1}^{N} c_{k} \beta(0, k, j)=c_{j},
$$

by (ii) of Proposition 2.1. Consequently,

$$
c_{j}=b\left(w_{j}(s)\right),
$$

and the solution of the system (2.17) is given as follows:

$$
\tilde{V}_{j}^{s}(\tilde{t})=\sum_{k=1}^{N} b\left(w_{k}(s)\right) \beta(\tilde{t}, k, j)
$$

or, in terms of $t$,

$$
\tilde{V}_{j}^{s}(t)=\sum_{k=1}^{N} b\left(w_{k}(s)\right) \beta\left(\int_{0}^{t-s} D(z) d z, k, j\right)
$$

Hence,

$$
u_{j}(t, t-s)=V_{j}^{s}(t)=e^{-\int_{0}^{t-s} d(z) d z} \sum_{k=1}^{N} b\left(w_{k}(s)\right) \beta\left(\int_{0}^{t-s} D(z) d z, k, j\right) .
$$

Recalling that $s \geq 0$ we deduce that, for $t \geq r$,

$$
u_{j}(t, r)=\mu \sum_{k=1}^{N} b\left(w_{k}(t-r)\right) \beta(\alpha, k, j),
$$

where $b\left(w_{k}(t)\right)$ is the birth function introduced above, and $\mu$ and $\alpha$ are given by (2.9). Thus, the system for $w_{j}$ has the form (2.10) for $t \geq r$.

3. Positivity of solutions. In the previous sections we derived the reduced model for $w_{j}(t)$ and proved various properties of $\beta(t, k, j)$. Using the fact that $\beta(t, k, j)>0$ for all $t>0$ we shall now prove a positivity-preserving property for system (2.10).

The result which we shall prove in this section is for the system consisting of the differential equations (2.10) for $t>0$, supplemented by the initial data (2.11). As noted previously, the reduced system (2.10) is actually only valid for $t \geq r$. For $0<t<r$ another nonautonomous system applies and governs $w_{j}(t)$. Thus there 
is a subtle relationship between the original problem (2.1) and the reduced problem (2.10) as regards positivity. This issue is discussed in detail in [2] for systems without diffusion, and the issues concerning positivity are basically the same for systems with and without diffusion.

First, we consider the initial value problem

$$
\frac{d \mathbf{v}(t)}{d t}=D A \mathbf{v}(t)-d \mathbf{v}(t)+\mathbf{h}(t),
$$

where $\mathbf{v}(t)=\left(v_{1}(t), \ldots, v_{N}(t)\right)^{T}$ and $\mathbf{h}(t)=\left(h_{1}(t), \ldots, h_{N}(t)\right)^{T}$, subject to

$$
v_{j}(0)=c_{j}, c_{j} \in \mathbb{R}, j=1, \ldots, N .
$$

From the definition of $\beta(t, k, j)$ it is easy to check that the solution to this problem is

$v_{j}(t)=e^{-d t} \sum_{k=1}^{N} \beta(D t, k, j) c_{k}+\sum_{k=1}^{N} \int_{0}^{t} e^{-d(t-s)} \beta(D(t-s), k, j) h_{k}(s) d s, j=1, \ldots, N$.

Therefore if $c_{j} \geq 0$ and $h_{j}(t) \geq 0$ for all $t \geq 0$ and $j=1, \ldots, N$, then, since $\beta(t, k, j) \geq$ 0 , we have $v_{j}(t) \geq 0$ for all $j=1, \ldots, N$ and $t \geq 0$. We shall now prove that the solutions of (2.10) enjoy positivity-preserving properties analogous to results that can be proved using the strong maximum principle in the case of continuous space.

TheOREm 3.1. Let $b(0)=0$ and $b(w)>0$ when $w>0$, and let $\left\{w_{j}\right\}$ be the solution of system (2.10), $t>0$, corresponding to the initial data $w_{j}(s)=w_{j}^{0}(s)$, $s \in[-r, 0]$. If $w_{j}^{0}(s) \geq 0$ for all $j=1, \ldots, N$ and $s \in[-r, 0]$, then $w_{j}(t) \geq 0$ for all $j=1, \ldots, N$ and $t \geq 0$.

Also, if $w_{j}^{0}(s) \not \equiv 0$ on $(s, j) \in[-r, 0] \times\{1,2, \ldots, N\}$, then $w_{j}(t)>0$ for all $j=1, \ldots, N$ and $t \geq r$.

Proof. We first prove the nonnegativity property $w_{j}(t) \geq 0$. This is achieved in steps; we first prove the result for $t \in[0, r]$. Applying (3.2) to system (2.10) with

$$
h_{j}(t)=\mu \sum_{k=1}^{N} \beta(\alpha, k, j) b\left(w_{k}(t-r)\right)
$$

gives, for $t \in[0, r]$,

$$
\begin{aligned}
w_{j}(t)= & e^{-d_{m} t} \sum_{k=1}^{N} \beta\left(D_{m} t, k, j\right) w_{k}(0) \\
& +\mu \sum_{k=1}^{N} \int_{0}^{t} e^{-d_{m}(t-s)} \beta\left(D_{m}(t-s), k, j\right) \sum_{l=1}^{N} \beta(\alpha, l, k) b\left(w_{l}(s-r)\right) d s .
\end{aligned}
$$

Now $t \in[0, r]$ so the right-hand side of the above expression refers only to the initial data for $w_{j}(t)$, which is nonnegative by hypothesis. Since $\beta$ is nonnegative also, we conclude that $w_{j}(t) \geq 0$ for $t \in[0, r]$. On the interval $t \in[r, 2 r]$ an expression analogous to $(3.3)$ can easily be found, referring in its right-hand side to $w_{j}(\cdot)$ only at times between 0 and $r$, on which interval we have just shown nonnegativity. Therefore, $w_{j}(t) \geq 0$ for $t \in[r, 2 r]$. This argument can be continued indefinitely, and so we have shown $w_{j}(t) \geq 0$ for all $t \geq 0$. 
We now prove the second part of the theorem: strict positivity of $w_{j}(t)$ for all $t \geq r$, provided the initial data is not identically zero. From (3.3) we can infer that

$$
w_{j}(r)>0 \quad \text { for all } j=1, \ldots, N .
$$

Indeed, if we had $w_{j}(r)=0$, then, since $\beta(t, k, j)>0$ for $t>0$ and since $b(\cdot)$ is positive definite, it would follow that $w_{j}(s) \equiv 0$ on $(s, j) \in[-r, 0] \times\{1,2, \ldots, N\}$, contrary to hypothesis. For $t \geq r$ an expression for $w_{j}(t)$ similar to (3.3) can be found, and from this expression we can infer that, for $t \geq r$,

$$
w_{j}(t) \geq e^{-d_{m}(t-r)} \sum_{k=1}^{N} \beta\left(D_{m}(t-r), k, j\right) w_{k}(r)>0
$$

for all $j=1, \ldots, N$. The proof of the theorem is complete.

4. Comparison principle: Monotone birth functions. In this section we shall prove a comparison theorem for system (2.10) for the case when the birth function is increasing, and we shall use it to prove that in this case if a positive uniform equilibrium solution exists, then it is globally stable. Theorem 4.1 below can also be established using results in Chapter 5 of Smith [6] (indeed, for increasing birth functions system (2.10) satisfies the quasimonotone condition on page 78 of [6], and (2.10) constitutes a cooperative and irreducible system of functional differential equations). However, we will include a self-contained proof of our Theorem 4.1 here because, as will become clear later, a detailed understanding of the monotone case throws much light on how one can approach the case of a nonmonotone birth function which we do later in Theorem 5.1.

THEOREM 4.1. Let the birth function $b(w)$ be increasing and differentiable for all $w \geq 0$ and let $\beta(t, k, j)$ be given by $(2.6)$. Let $\overline{\mathbf{w}}(t)=\left(\bar{w}_{1}(t), \ldots, \bar{w}_{N}(t)\right)^{T}$ and $\hat{\mathbf{w}}(t)=\left(\hat{w}_{1}(t), \ldots, \hat{w}_{N}(t)\right)^{T}$ be such that

$$
\begin{aligned}
& \frac{d \overline{\mathbf{w}}(t)}{d t}-D_{m} A \overline{\mathbf{w}}(t)+d_{m} \overline{\mathbf{w}}(t)-\mu \sum_{k=1}^{N} b\left(\bar{w}_{k}(t-r)\right)\left(\begin{array}{l}
\beta(\alpha, k, 1) \\
\beta(\alpha, k, 2) \\
\vdots \\
\beta(\alpha, k, N-1) \\
\beta(\alpha, k, N)
\end{array}\right) \\
& \geq \frac{d \hat{\mathbf{w}}(t)}{d t}-D_{m} A \hat{\mathbf{w}}(t)+d_{m} \hat{\mathbf{w}}(t)-\mu \sum_{k=1}^{N} b\left(\hat{w}_{k}(t-r)\right)\left(\begin{array}{l}
\beta(\alpha, k, 1) \\
\beta(\alpha, k, 2) \\
\vdots \\
\beta(\alpha, k, N-1) \\
\beta(\alpha, k, N)
\end{array}\right)
\end{aligned}
$$

for $t>0$ and

$$
\bar{w}_{j}(s) \geq \hat{w}_{j}(s), \quad j=1, \ldots, N, \quad s \in[-r, 0] .
$$

Then $\bar{w}_{j}(t) \geq \hat{w}_{j}(t)$ for all $t>0$ and $j=1, \ldots, N$. 
Proof. Let $\mathbf{W}(t)$ be the vector with components $W_{j}(t):=\bar{w}_{j}(t)-\hat{w}_{j}(t)$. Then (4.1) can be rewritten as

$$
\begin{aligned}
& \frac{d \mathbf{W}(t)}{d t}-D_{m} A \mathbf{W}(t)+d_{m} \mathbf{W}(t) \\
& -\mu \sum_{k=1}^{N}\left[b\left(\bar{w}_{k}(t-r)\right)-b\left(\hat{w}_{k}(t-r)\right)\right]\left(\begin{array}{l}
\beta(\alpha, k, 1) \\
\beta(\alpha, k, 2) \\
\vdots \\
\beta(\alpha, k, N-1) \\
\beta(\alpha, k, N)
\end{array}\right) \geq 0 .
\end{aligned}
$$

Applying the mean value theorem to the last term in the left-hand side of the above inequality we obtain

$$
\frac{d \mathbf{W}(t)}{d t}-D_{m} A \mathbf{W}(t)+d_{m} \mathbf{W}(t)-\mu \sum_{k=1}^{N} b^{\prime}\left(\theta_{k}(t-r)\right) W_{k}(t-r)\left(\begin{array}{l}
\beta(\alpha, k, 1) \\
\beta(\alpha, k, 2) \\
\vdots \\
\beta(\alpha, k, N-1) \\
\beta(\alpha, k, N)
\end{array}\right) \geq 0
$$

where $\theta_{k}(t)$ is between $\bar{w}_{k}(t)$ and $\hat{w}_{k}(t), k=1, \ldots, N$. By hypothesis $\bar{w}_{j}(s) \geq \hat{w}_{j}(s)$ for $s \in[-r, 0]$, so $W_{j}(s) \geq 0$ for $s \in[-r, 0]$. To prove the theorem we need to show that $W_{j}(t) \geq 0$ for all $t>0$, and as a first step we shall prove this fact for $t \in(0, r]$. For $t \in(0, r]$,

$$
f_{j}(t):=\mu \sum_{k=1}^{N} b^{\prime}\left(\theta_{k}(t-r)\right) W_{k}(t-r) \beta(\alpha, k, j) \geq 0 .
$$

Inequality (4.2) becomes

$$
\frac{d \mathbf{W}(t)}{d t}-D_{m} A \mathbf{W}(t)+d_{m} \mathbf{W}(t) \geq \mathbf{f}(t),
$$

where $\mathbf{f}(t)=\left(f_{1}(t), \ldots, f_{N}(t)\right)^{T}$. We claim that $W_{j}(t) \geq 0$ for all $j=1, \ldots, N$ and $t \in[0, r]$. Suppose this is false, i.e., that $W_{j}(t)$ goes negative. Then $W_{j}(t)$ must attain a negative minimum on the set $(t, j) \in[0, r] \times\{1,2, \ldots, N\}$. Let this happen at time $t^{*}$ and at the node $j^{*}$. Since $W_{j}(0) \geq 0$ we must have $t^{*}>0$, but it is possible that $t^{*}=r$. In any case,

$$
\frac{d W_{j^{*}}\left(t^{*}\right)}{d t} \leq 0,
$$

and, of course, $W_{j^{*}}\left(t^{*}\right)<0$. Also, if $j^{*}$ is an "interior" node, then

$$
W_{j^{*}-1}\left(t^{*}\right)-2 W_{j^{*}}\left(t^{*}\right)+W_{j^{*}+1}\left(t^{*}\right) \geq 0
$$

while if $j^{*}=1$, then $-W_{1}\left(t^{*}\right)+W_{2}\left(t^{*}\right) \geq 0$ and similarly if $j^{*}=N$. Extracting the $j^{*}$ th component of (4.3) and evaluating it at time $t^{*}$ gives

$$
\underbrace{\frac{d W_{j^{*}}\left(t^{*}\right)}{d t}-\left(j^{*} \text { th component of } D_{m} \text { term }\right)}_{\leq 0}+\underbrace{d_{m} W_{j^{*}}\left(t^{*}\right)}_{<0} \geq \underbrace{f_{j^{*}}\left(t^{*}\right)}_{\geq 0}
$$


which is a contradiction. Thus $W_{j}(t) \geq 0$ for all $j=1, \ldots, N$ and $t \in[0, r]$. Repeating this argument establishes that $W_{j}(t) \geq 0$ for $t \in[r, 2 r]$ and the argument can be continued to include all positive times. The proof of the theorem is complete.

4.1. Convergence to equilibrium. In this section we will prove that if the birth function $b(w)$ is increasing and is such that there exists a uniform equilibrium solution $w^{*}$ (independent of both $j$ and $t$ ) to system (2.10) and is biologically realistic, then solutions of (2.10) approach the equilibrium $w^{*}$. Note first that a uniform equilibrium state $w^{*}$ must necessarily satisfy

$$
\mu b\left(w^{*}\right)=d_{m} w^{*} .
$$

We will prove the following theorem.

THEOREM 4.2. In system (2.10) let the birth function $b(w)$ satisfy $b(0)=0$ and be an increasing differentiable function for all $w \geq 0$. Assume there exists $w^{*}>0$ such that $\mu b(w)>d_{m} w$ when $0<w<w^{*}$ and $\mu b(w)<d_{m} w$ when $w>w^{*}$. Assume further that, in $(2.11), w_{j}^{0}(s) \not \equiv 0$ on $(s, j) \in[-r, 0] \times\{1,2, \ldots, N\}$. Then the solution $w_{j}(t)$ of (2.10) for $t>0$, subject to (2.11), satisfies $w_{j}(t) \rightarrow w^{*}$ as $t \rightarrow \infty$, for each $j=1,2, \ldots, N$.

Proof. To prove this theorem we shall use Theorem 4.1. More specifically, we shall show using Theorem 4.1 that the solution $w_{j}(t)$ of (2.10) subject to (2.11) can be bounded above and below by solutions of (2.10) that are functions of $t$ only.

Indeed, if we denote by $w(t)$ any solution of the scalar equation

$$
\frac{d w(t)}{d t}=\mu b(w(t-r))-d_{m} w(t),
$$

then the function

$$
\left(w_{1}(t), w_{2}(t), \ldots, w_{N}(t)\right):=(w(t), w(t), \ldots, w(t))
$$

satisfies (2.10). Two applications of Theorem 4.1 are required. In the first, we choose $\hat{w}_{j}(t)$ to be the solution $w_{j}(t)$ of (2.10) subject to $(2.11)$ and $\bar{w}_{j}(t)=\bar{w}(t)$ for each $j$, where $\bar{w}(t)$ satisfies

$$
\begin{aligned}
& \frac{d \bar{w}(t)}{d t}=\mu b(\bar{w}(t-r))-d_{m} \bar{w}(t), \\
& \bar{w}(s)=\max \left\{w_{j}^{0}(s), j=1,2, \ldots, N\right\} \quad \text { for } s \in[-r, 0] .
\end{aligned}
$$

Then Theorem 4.1 yields

$$
w_{j}(t) \leq \bar{w}(t), \quad t>0, \quad j=1,2, \ldots, N .
$$

For the second application of Theorem 4.1 the most obvious choices are to take $\bar{w}_{j}(t)$ as the solution $w_{j}(t)$ of (2.10) subject to (2.11) and, for each $j, \hat{w}_{j}(t)=\hat{w}(t)$ where $\hat{w}(t)$ satisfies

$$
\begin{aligned}
& \frac{d \hat{w}(t)}{d t}=\mu b(\hat{w}(t-r))-d_{m} \hat{w}(t), \\
& \hat{w}(s)=\min \left\{w_{j}^{0}(s), j=1,2, \ldots, N\right\} \quad \text { for } s \in[-r, 0]
\end{aligned}
$$

so that

$$
w_{j}(t) \geq \hat{w}(t), \quad t>0, \quad j=1,2, \ldots, N
$$


but this presents a possible problem in that $\hat{w}(s)$ could be zero on all of $s \in[-r, 0]$ without violating the assumption $w_{j}^{0}(s) \not \equiv 0$ on $(s, j) \in[-r, 0] \times\{1,2, \ldots, N\}$ (e.g., if $w_{j}^{0}(s)$ were zero for all $s$ on one particular node) in which case $\hat{w}(t)$ would be zero for all $t>0$, which is not helpful for us. The way round this difficulty is to remember that we showed earlier (Theorem 3.1) that $w_{j}(t)>0$ for all $t \geq r$. Consider the initial value problem starting at time $t=2 r$ and consisting of equation (2.10) for $t>2 r$, with initial data taken to be the solution $w_{j}(t), t \in[r, 2 r]$, of the original problem. The solution of this new initial value problem for $t>2 r$ is clearly the same as the solution $w_{j}(t)$ of the original problem, but the new problem has strictly positive initial data. This means that, without loss of generality, the minimum in (4.7) can be assumed to be strictly positive for all $s \in[-r, 0]$.

To complete the proof of Theorem 4.2 it therefore suffices to prove that every solution of the scalar ODE (4.5) such that $w(s)>0$ for all $s \in[-r, 0]$ will satisfy $w(t) \rightarrow w^{*}$, if the hypotheses on the parameters and the function $b(w)$ are satisfied. This follows immediately from Theorem 9.1 on page 159 of the book by Kuang [9]. Therefore, the proof of Theorem 4.2 is complete.

5. Nonmonotone birth functions. Of considerable interest to ecologists, is the case of a birth function $b(w)$ which is increasing up to a certain value of $w$ and decreasing thereafter (for example, a function qualitatively resembling $b(w)=P w e^{-A w}$ ). Such birth functions are important in modelling certain insect populations in which the birth rate is observed to be roughly proportional to the number of adults if the number of adults is small, but effectively zero if the number of adults is large, since competition for resources then becomes so intense that the adults require all their resources for their own maintenance. The aims of this section are to establish a comparison principle that works for very general birth functions, and then to use the comparison principle to prove convergence theorems in the case when the birth function qualitatively resembles $b(w)=P w e^{-A w}$.

THEOREM 5.1. Let the birth function $b(w)$ be a differentiable function for all $w \geq 0$ and satisfy $b(0)=0, b(w)>0$ when $w>0$. Let $\hat{w}$ and $\bar{w}$ be a pair of sub-and supersolutions for (2.10), (2.11), i.e., a pair of functions satisfying

(i) $\hat{w}_{j}(t) \leq \bar{w}_{j}(t)$ for all $t \in[-r, \infty), j=1,2, \ldots, N$;

(ii) letting $\overline{\mathbf{w}}(t)=\left(\bar{w}_{1}(t), \ldots, \bar{w}_{N}(t)\right)^{T}$ and $\hat{\mathbf{w}}(t)=\left(\hat{w}_{1}(t), \ldots, \hat{w}_{N}(t)\right)^{T}$ for $t>0$ and $j=1,2, \ldots, N$,

$$
\frac{d \hat{\mathbf{w}}(t)}{d t} \leq D_{m} A \hat{\mathbf{w}}(t)-d_{m} \hat{\mathbf{w}}(t)+\mu \sum_{k=1}^{N} b\left(\varphi_{k}(t-r)\right)\left(\begin{array}{l}
\beta(\alpha, k, 1) \\
\beta(\alpha, k, 2) \\
\vdots \\
\beta(\alpha, k, N-1) \\
\beta(\alpha, k, N)
\end{array}\right)
$$

and

$$
\frac{d \overline{\mathbf{w}}(t)}{d t} \geq D_{m} A \overline{\mathbf{w}}(t)-d_{m} \overline{\mathbf{w}}(t)+\mu \sum_{k=1}^{N} b\left(\varphi_{k}(t-r)\right)\left(\begin{array}{l}
\beta(\alpha, k, 1) \\
\beta(\alpha, k, 2) \\
\vdots \\
\beta(\alpha, k, N-1) \\
\beta(\alpha, k, N)
\end{array}\right)
$$


for all functions $\varphi_{j}(t)$ such that $\hat{w}_{j}(t) \leq \varphi_{j}(t) \leq \bar{w}_{j}(t), t \in[-r, \infty), j=$ $1,2, \ldots, N$;

(iii) $\hat{w}_{j}(s) \leq w_{j}^{0}(s) \leq \bar{w}_{j}(s), s \in[-r, 0], j=1,2, \ldots, N$, where $w_{j}^{0}(s)$ is the initial data for $(2.10)$.

Then the solution $w_{j}(t)$ of $(2.10),(2.11)$ satisfies

$$
\hat{w}_{j}(t) \leq w_{j}(t) \leq \bar{w}_{j}(t) \quad \text { for all } t>0, j=1,2, \ldots, N .
$$

Proof. Using (2.10), inequality (5.1) can be rewritten as

$$
\begin{aligned}
& \frac{d \hat{\mathbf{w}}(t)}{d t}-D_{m} A \hat{\mathbf{w}}(t)+d_{m} \hat{\mathbf{w}}(t)-\mu \sum_{k=1}^{N} b\left(\varphi_{k}(t-r)\right)\left(\begin{array}{l}
\beta(\alpha, k, 1) \\
\beta(\alpha, k, 2) \\
\vdots \\
\beta(\alpha, k, N-1) \\
\beta(\alpha, k, N)
\end{array}\right) \\
& \leq \frac{d \mathbf{w}(t)}{d t}-D_{m} A \mathbf{w}(t)+d_{m} \mathbf{w}(t)-\mu \sum_{k=1}^{N} b\left(w_{k}(t-r)\right)\left(\begin{array}{l}
\beta(\alpha, k, 1) \\
\beta(\alpha, k, 2) \\
\vdots \\
\beta(\alpha, k, N-1) \\
\beta(\alpha, k, N)
\end{array}\right) .
\end{aligned}
$$

Define $W_{j}(t)=w_{j}(t)-\hat{w}_{j}(t)$ and $\mathbf{W}(t)$ to be the vector with components $W_{j}(t)$. Then (5.3) becomes

$$
\begin{array}{r}
\frac{d \mathbf{W}(t)}{d t}-D_{m} A \mathbf{W}(t)+d_{m} \mathbf{W}(t)-\mu \sum_{k=1}^{N}\left[b\left(w_{k}(t-r)\right)\right. \\
\left.-b\left(\varphi_{k}(t-r)\right)\right]\left(\begin{array}{l}
\beta(\alpha, k, 1) \\
\beta(\alpha, k, 2) \\
\vdots \\
\beta(\alpha, k, N-1) \\
\beta(\alpha, k, N)
\end{array}\right) \geq 0
\end{array}
$$

and by hypothesis this holds for all $\varphi_{j}(t)$ such that $\hat{w}_{j}(t) \leq \varphi_{j}(t) \leq \bar{w}_{j}(t), t \in[-r, \infty)$, $j=1,2, \ldots, N$.

We need to prove that $W_{j}(t) \geq 0$ for all $t>0$ and all $j$, and we shall first prove this conclusion for $t \in(0, r]$. In inequality (5.4), for each $j$ choose $\varphi_{j}(t)$ to be any function between $\hat{w}_{j}(t)$ and $\bar{w}_{j}(t), t \in[-r, \infty)$, which is such that $\varphi_{j}(s)=w_{j}^{0}(s)$ when $s \in[-r, 0]$. From this choice for $\varphi_{j}(t)$ we infer that, for $t \in(0, r]$ only, the last term in the left-hand side of inequality (5.4) is zero, so that the inequality holds for $t \in(0, r]$ with just the first three terms in the left-hand side. The proof that $W_{j}(t) \geq 0$ for $t \in(0, r]$ then proceeds the same way as in the proof of Theorem 4.1 because our inequality is the same as (4.3) in the case when the functions $f_{i}(t)$ of the latter are zero.

Proving that $w_{j}(t) \leq \bar{w}_{j}(t)$ for $t \in(0, r]$ and all $j$ is similar. Thus

$$
\hat{w}_{j}(t) \leq w_{j}(t) \leq \bar{w}_{j}(t) \quad \text { for } t \in(0, r], j=1,2, \ldots, N .
$$




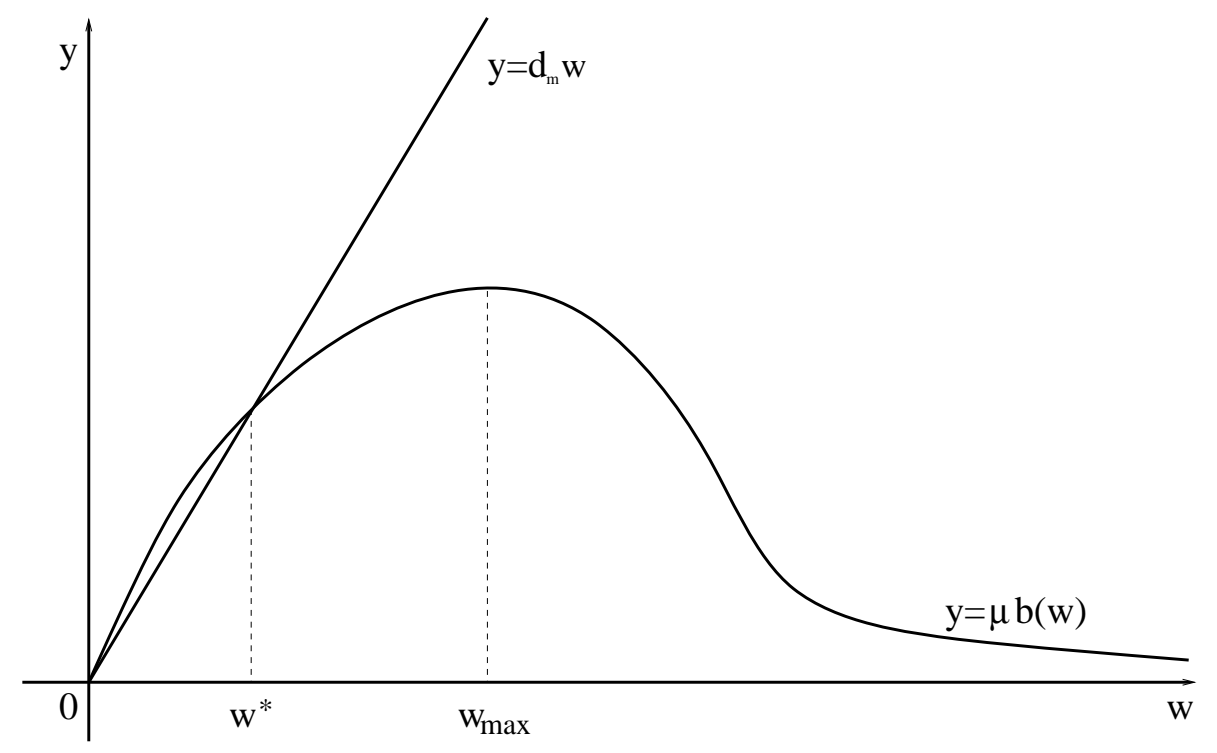

FIG. 1. Graphical depiction of the situation in which Theorem 5.2 holds. In particular, the equilibrium $w^{*}$ has to satisfy $0<w^{*}<w_{\max }$.

Proving that $W_{j}(t)=w_{j}(t)-\hat{w}_{j}(t) \geq 0$ for $t \in(r, 2 r]$ is similar. Inequality (5.4) still holds for all $t>0$ and in particular for $t \in(r, 2 r]$. This time, we choose $\varphi_{j}(t)$ to be any function between $\hat{w}_{j}(t)$ and $\bar{w}_{j}(t), t \in[-r, \infty)$, which is such that $\varphi_{j}(t)=w_{j}(t)$ when $t \in[0, r]$. This choice furnishes for us inequality (5.4), on $t \in(r, 2 r]$ only, but without the term involving summation. We thus conclude that $W_{j}(t) \geq 0$ for $t \in(r, 2 r]$ and it is clear how to continue the proof.

Remark. A comparison theorem similar to Theorem 5.1 was proved for the case of continuous space by Redlinger [5].

5.1. Convergence to equilibrium when $\boldsymbol{w}^{*}<\boldsymbol{w}_{\max }$. We will use Theorem 5.1 to establish, essentially, that if $b(w)$ qualitatively resembles $P w e^{-A w}$ and if a nonzero equilibrium of (2.10) exists, is unique, and is in the interval of $w$ for which $b(w)$ is increasing, then the equilibrium is globally stable as a solution of (2.10).

THEOREM 5.2. In system (2.10) let the birth function $b(w)$ satisfy $b(0)=0$ and $b(w)>0$ when $w>0$. Also, let $b(w)$ be increasing for $0<w<w_{\max }$, with $b^{\prime}\left(w_{\max }\right)=0$, and decreasing for $w>w_{\max }$. Assume further that there exists $w^{*}>0$ such that $\mu b(w)>d_{m} w$ when $0<w<w^{*}$ and $\mu b(w)<d_{m} w$ when $w>w^{*}$, and assume that $w^{*}<w_{\max }$.

Then, if $w_{j}^{0}(s) \not \equiv 0$ on $(s, j) \in[-r, 0] \times\{1,2, \ldots, N\}$, the solution $w_{j}(t)$ of $(2.10)$ for $t>0$, subject to $(2.11)$, satisfies $w_{j}(t) \rightarrow w^{*}$ as $t \rightarrow \infty$, for each $j=1,2, \ldots, N$.

Remark. The situation we have in mind is shown in Figure 1.

Proof of Theorem 5.2. Let $\hat{w}_{j}(t)=0$ and $\bar{w}_{j}(t)=v(t)$ for each $j=1,2, \ldots, N$, where $v(t)$ is the solution of

$$
\begin{aligned}
& \frac{d v(t)}{d t}=\mu b\left(w_{\max }\right)-d_{m} v(t), \\
& v(s)=\max \left\{w_{j}^{0}(s), j=1,2, \ldots, N\right\}, \quad s \in[-r, 0] .
\end{aligned}
$$


It is easily seen that $\hat{w}_{j}(t)$ and $\bar{w}_{j}(t)$ are a pair of sub- and supersolutions. Thus, by Theorem 5.1,

$$
0 \leq w_{j}(t) \leq v(t) \quad \text { for all } t>0, j=1, \ldots, N \text {. }
$$

Thus

$$
\limsup _{t \rightarrow \infty} \max _{j \in\{1,2, \ldots, N\}} w_{j}(t) \leq \lim _{t \rightarrow \infty} v(t)=\frac{\mu b\left(w_{\max }\right)}{d_{m}} .
$$

Under the hypotheses it can be shown that

$$
w^{*}<\frac{\mu b\left(w_{\max }\right)}{d_{m}}<w_{\max } .
$$

Choose $\epsilon>0$ sufficiently small such that

$$
\frac{\mu b\left(w_{\max }\right)}{d_{m}}+\epsilon<w_{\max } .
$$

There exists a time $T>0$ such that, for all $t>T$ and all $j$,

$$
w_{j}(t) \leq \frac{\mu b\left(w_{\max }\right)}{d_{m}}+\epsilon<w_{\max } .
$$

Then as soon as $t$ exceeds $T+r$ there is effectively no record, as far as system (2.10) is concerned, of the solution $w_{j}(t)$ ever having taken values outside the interval $\left[0, w_{\text {max }}\right]$. From this point on the analysis proceeds as if the birth function were increasing for all $w$, and therefore it follows from Theorem 4.2 that the solution converges to $w^{*}$. The proof is complete.

5.2. Convergence to equilibrium when $\boldsymbol{w}^{*}>\boldsymbol{w}_{\max }$. This section will show that if $w^{*}>w_{\max }$, then solutions of (2.10), (2.11) will still converge to $w^{*}$ if additional conditions hold. These additional conditions will hold if $w^{*}$ is not too much larger than $w_{\max }$.

THEOREM 5.3. In system (2.10) let the birth function $b(w)$ satisfy $b(0)=0$ and $b(w)>0$ when $w>0$. Also, let $b(w)$ be increasing for $0<w<w_{\max }$, with $b^{\prime}\left(w_{\max }\right)=0$, and decreasing for $w>w_{\max }$. Assume further that there exists $w^{*}>0$ such that $\mu b(w)>d_{m} w$ when $0<w<w^{*}$ and $\mu b(w)<d_{m} w$ when $w>w^{*}$. Assume that $w^{*}>w_{\max }$ and that

$$
\frac{1}{d_{m}} \mu b\left(\frac{\mu b\left(w_{\max }\right)}{d_{m}}\right)>w_{\max } .
$$

Furthermore, we assume that

$$
\left(d_{m}+\bar{f}\right) r<1,
$$

where

$$
\bar{f}=\mu \max \left\{\left|b^{\prime}(w)\right|, \quad w \in[\underline{w}, \bar{w}]\right\}
$$

with $\bar{w}=\left(\mu / d_{m}\right) b\left(w_{\max }\right)$ and $\underline{w}=\left.b^{-1}\left(b\left(w^{*}\right)\right)\right|_{\left[0, w_{\max }\right]}$. Assume further that

$$
\frac{\mu}{d_{m}} b^{\prime}\left(w^{*}\right)>-1
$$




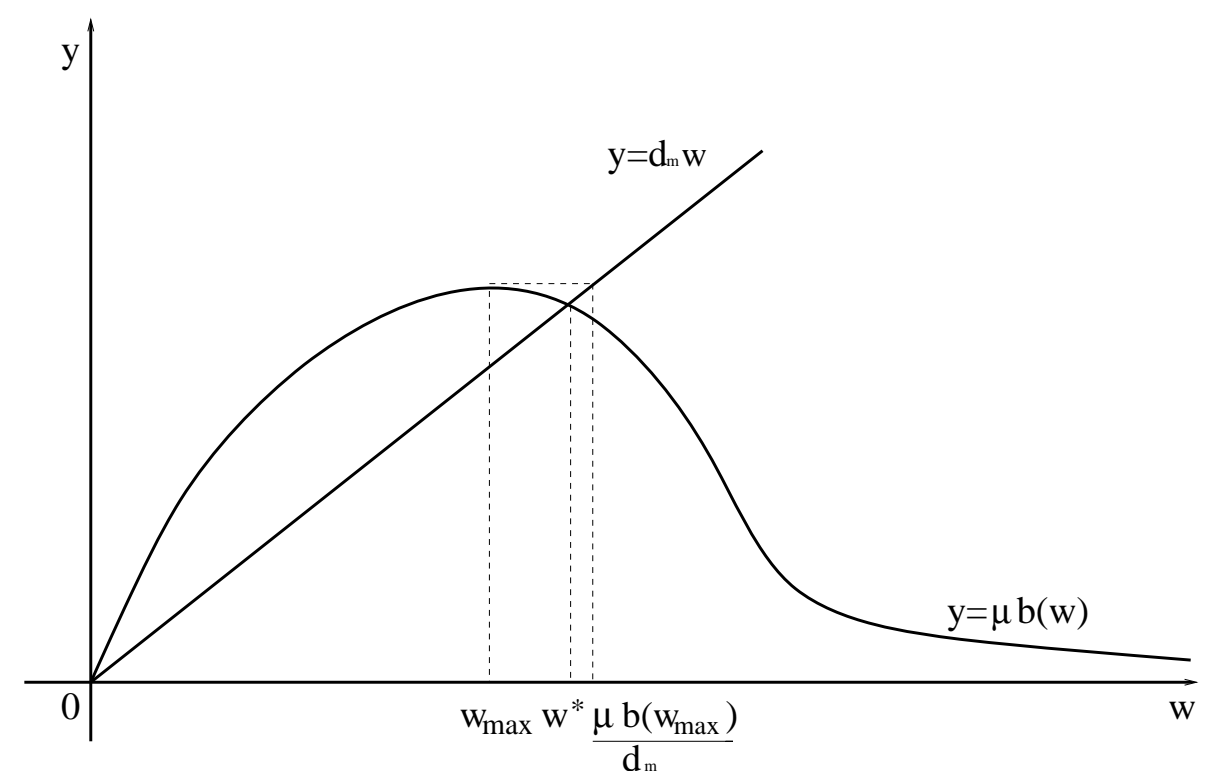

FIG. 2. Graphical depiction of the situation in which Theorem 5.3 holds. In particular, the equilibrium $w^{*}$ has to satisfy $w^{*}>w_{\max }$.

Then, if $w_{j}^{0}(s) \not \equiv 0$ on $(s, j) \in[-r, 0] \times\{1,2, \ldots, N\}$, the solution $w_{j}(t)$ of $(2.10)$ for $t>0$, subject to (2.11) satisfies $w_{j}(t) \rightarrow w^{*}$ as $t \rightarrow \infty$, for each $j=1,2, \ldots, N$.

Remarks. It is natural to question whether these hypotheses can be satisfied. The graph shown in Figure 2 shows that they can, and also suggests that the hypotheses are likely to be satisfied only when $w^{*}$ is not too much greater than $w_{\max }$.

The notation $\left.b^{-1}\left(b\left(w^{*}\right)\right)\right|_{\left[0, w_{\max }\right]}$ requires some explaining. Under the assumptions on $b(w), b^{-1}(w)$ will, if defined, have in general two values. Thus $b^{-1}\left(b\left(w^{*}\right)\right)$ is either $w^{*}$ or a value in $\left[0, w_{\max }\right]$ and $\left.b^{-1}\left(b\left(w^{*}\right)\right)\right|_{\left[0, w_{\max }\right]}$ means the latter value.

Proof of Theorem 5.3. Let us define

$$
\begin{aligned}
& w_{\max }^{0}=\max \left\{w^{*}, \max \left\{w_{j}^{0}(s), j=1,2, \ldots, N, \quad s \in[-r, 0]\right\}\right\}, \\
& w_{\min }^{0}=\min \left\{w^{*}, \min \left\{w_{j}^{0}(s), j=1,2, \ldots, N, \quad s \in[-r, 0]\right\}\right\} .
\end{aligned}
$$

We can assume without loss of generality that $w_{\min }^{0}>0$ (this can be justified similarly to the proof of Theorem 4.2).

The proof begins with the observation that $\left(\hat{w}_{j}(t), \bar{w}_{j}(t)\right)=\left(0, V_{1}(t)\right)$ is a sub/ supersolution pair for $(2.10),(2.11)$, where $V_{1}(t)$ satisfies

$$
\begin{aligned}
& \frac{d V_{1}(t)}{d t}=\mu b\left(w_{\max }\right)-d_{m} V_{1}(t), \\
& V_{1}(s)=w_{\max }^{0}, s \in[-r, 0] .
\end{aligned}
$$

Therefore,

$$
\limsup _{t \rightarrow \infty} \max _{j \in\{1,2, \ldots, N\}} w_{j}(t) \leq \lim _{t \rightarrow \infty} V_{1}(t)=\frac{\mu b\left(w_{\max }\right)}{d_{m}} .
$$

Next it is easy to see that $\left(v_{1}, V_{1}\right)$ is a sub/supersolution pair, where $V_{1}$ is the above 
function and $v_{1}$ satisfies

$$
\begin{aligned}
& \frac{d v_{1}}{d t}=\mu \min \left\{b\left(v_{1}(t-r)\right), b\left(V_{1}(t-r)\right)\right\}-d_{m} v_{1}(t), \\
& v_{1}(s)=w_{\min }^{0}, s \in[-r, 0] .
\end{aligned}
$$

Inequality (5.7) assures us that the solution of

$$
\frac{d v}{d t}=\mu b(v(t-r))-d_{m} v(t)
$$

with positive initial data satisfies $\lim _{t \rightarrow \infty} v(t)=w^{*}$. This follows from Theorem 9.5 in Kuang (see [9, page 165]). On the other hand, any solution of

$$
\frac{d v}{d t}=\mu b\left(V_{1}(t-r)\right)-d_{m} v(t)
$$

satisfies

$$
\lim _{t \rightarrow \infty} v(t)=\frac{\mu}{d_{m}} b\left(\frac{\mu b\left(w_{\max }\right)}{d_{m}}\right)
$$

Therefore the solution $v_{1}(t)$ of (5.9) tends, as $t \rightarrow \infty$, to either $w^{*}$ or $\frac{\mu}{d_{m}} b\left(\frac{\mu b\left(w_{\max }\right)}{d_{m}}\right)$ and so our proof proceeds by considering two cases.

Case $1 . \lim _{t \rightarrow \infty} v_{1}(t)=w^{*}$. In this case we obtain immediately that

$$
\liminf _{t \rightarrow \infty} \min _{j \in\{1,2, \ldots, N\}} w_{j}(t) \geq w^{*} .
$$

It can be shown similarly that

$$
\limsup _{t \rightarrow \infty} \max _{j \in\{1,2, \ldots, N\}} w_{j}(t) \leq w^{*},
$$

completing the proof of the theorem for Case 1 .

Case 2. $\lim _{t \rightarrow \infty} v_{1}(t)=\frac{\mu}{d_{m}} b\left(\frac{\mu b\left(w_{\max }\right)}{d_{m}}\right)$. What we initially get in this case is

$$
\liminf _{t \rightarrow \infty} \min _{j \in\{1,2, \ldots, N\}} w_{j}(t) \geq \frac{\mu}{d_{m}} b\left(\frac{\mu b\left(w_{\max }\right)}{d_{m}}\right),
$$

which, by (5.6), strictly exceeds $w_{\max }$. Therefore, for $t$ sufficiently large, $w_{j}(t)>w_{\max }$. Since the problem has finite delay, this further means that for $t$ sufficiently large there is no history of $w_{j}(t)$ ever having assumed values below $w_{\max }$, so that for the remainder of the proof $b(w)$ can be treated as decreasing in $w$. In fact we may shift the origin of time such as to assume, without loss of generality, that $w_{\min }^{0}>w_{\max }$. With this fact in mind, our proof now proceeds by successive refinement of pairs of sub/supersolutions.

In general, for each $n=2,3, \ldots$, let $\left(v_{n}, V_{n}\right)$ be defined by

$$
\begin{aligned}
& \frac{d v_{n}}{d t}=\mu b\left(V_{n-1}(t-r)\right)-d_{m} v_{n}, \\
& \frac{d V_{n}}{d t}=\mu b\left(v_{n-1}(t-r)\right)-d_{m} V_{n}
\end{aligned}
$$


with the initial conditions $V_{n}(s)=w_{\max }^{0}, s \in[-r, 0]$, and $v_{n}(s)=w_{\min }^{0}, s \in[-r, 0]$. We will show that $\left(v_{n}, V_{n}\right)$ is a sub/supersolution pair for each $n=2,3, \ldots$. According to Theorem 5.1, what we need to show is that

$$
b\left(V_{n-1}(t-r)\right) \leq \sum_{k=1}^{N} b\left(\varphi_{k}(t-r)\right) \beta(\alpha, k, j)
$$

whenever $\varphi_{j}(t)$ is such that $v_{n}(t) \leq \varphi_{j}(t) \leq V_{n}(t)$ for $j=1,2, \ldots, N$ and $t \in[-r, \infty)$. Since we are now working in an interval of $w$ in which $b(w)$ is decreasing, it is enough to establish that

$$
b\left(V_{n-1}(t-r)\right) \leq \sum_{k=1}^{N} b\left(V_{n}(t-r)\right) \beta(\alpha, k, j)=b\left(V_{n}(t-r)\right)
$$

and the latter is true if $V_{n}(t) \leq V_{n-1}(t)$ for each $n=2,3, \ldots$. Similarly, we need to show that $v_{n}(t) \geq v_{n-1}(t)$ for each $n$. In fact, we shall show by induction that

$$
v_{1}(t) \leq \cdots \leq v_{n-1}(t) \leq v_{n}(t)<w^{*}<V_{n}(t) \leq V_{n-1}(t) \leq \cdots \leq V_{1}(t) .
$$

To achieve this we assume (5.11) and prove that

$$
w^{*}<V_{n+1}(t) \leq V_{n}(t)
$$

and

$$
w^{*}>v_{n+1}(t) \geq v_{n}(t) .
$$

We will prove only (5.12). Now

$$
\begin{aligned}
\frac{d V_{n+1}(t)}{d t} & =\mu b\left(v_{n}(t-r)\right)-d_{m} V_{n+1}(t) \\
& \leq \mu b\left(v_{n-1}(t-r)\right)-d_{m} V_{n+1}(t) .
\end{aligned}
$$

Therefore

$$
\frac{d V_{n+1}(t)}{d t}+d_{m} V_{n+1}(t) \leq \frac{d V_{n}(t)}{d t}+d_{m} V_{n}(t)
$$

or, equivalently,

$$
\frac{d}{d t}\left(V_{n+1}-V_{n}\right)(t)+d_{m}\left(V_{n+1}-V_{n}\right)(t) \leq 0 .
$$

Therefore

$$
V_{n+1}(t)-V_{n}(t) \leq \underbrace{\left(V_{n+1}(0)-V_{n}(0)\right)}_{=0} e^{-d_{m} t}
$$

and so

$$
V_{n+1}(t) \leq V_{n}(t) .
$$

Also

$$
\begin{aligned}
\frac{d V_{n+1}(t)}{d t} & =\mu b\left(v_{n}(t-r)\right)-d_{m} V_{n+1}(t) \\
& \geq \mu b\left(w^{*}\right)-d_{m} V_{n+1}(t) \\
& =d_{m}\left(w^{*}-V_{n+1}\right)
\end{aligned}
$$


so that

$$
\frac{d}{d t}\left(V_{n+1}-w^{*}\right) \geq-d_{m}\left(V_{n+1}-w^{*}\right)
$$

Therefore,

$$
V_{n+1}(t)-w^{*} \geq \underbrace{\left(V_{n+1}(0)-w^{*}\right)}_{\geq 0} e^{-d_{m} t} \geq 0
$$

and so

$$
V_{n+1}(t) \geq w^{*}
$$

which establishes (5.12).

Denote $V_{n}^{*}=\lim _{t \rightarrow \infty} V_{n}(t)$ and $v_{n}^{*}=\lim _{t \rightarrow \infty} v_{n}(t)$. That these limits exist follows from (5.10) and an inductive argument. We know $v_{1}(t)$ and $V_{1}(t)$ approach limits as $t \rightarrow \infty$. There are theories of asymptotically autonomous differential equations which allow us to let $t \rightarrow \infty$ in system (5.10), with $n=2$, giving an autonomous system of differential equations from which it becomes clear that $v_{2}(t)$ and $V_{2}(t)$ approach limits as $t \rightarrow \infty$. The argument can be continued and we thus conclude the existence of the limits as $t \rightarrow \infty$ for each $n$. From (5.10) these limits satisfy

$$
\begin{aligned}
& \mu b\left(V_{n-1}^{*}\right)=d_{m} v_{n}^{*}, \\
& \mu b\left(v_{n-1}^{*}\right)=d_{m} V_{n}^{*} .
\end{aligned}
$$

We define $V^{*}=\lim _{n \rightarrow \infty} V_{n}^{*}$ and $v^{*}=\lim _{n \rightarrow \infty} v_{n}^{*}$; then (5.14) reduces to a limiting system

$$
\begin{aligned}
& \mu b\left(V^{*}\right)=d_{m} v^{*}, \\
& \mu b\left(v^{*}\right)=d_{m} V^{*} .
\end{aligned}
$$

These equations imply that $V^{*}=v^{*}=w^{*}$ (condition (5.8) assures us that they have no other solutions). Therefore, $\lim _{t \rightarrow \infty} w_{j}(t)=w^{*}$ and the proof of the theorem is complete.

6. Discussion. The main results in this paper are, first, the derivation of the model (2.10) itself, which is nontrivial and substantially different from the case of an infinite lattice considered previously in [8]. Second, the positivity-preservation properties of solutions which turn out to be analogous to results that can be proved using the strong maximum principle in the case of continuous space. Third, the comparison principle of section 4 for the case of increasing birth functions. Fourth, the use of this comparison principle to prove that the positive equilibrium of system (2.10), if feasible, is globally asymptotically stable for increasing birth functions. Fifth, in section 5, the comparison principle for the case of nonmonotone birth functions is proved. With the help of this principle we have shown that the nontrivial equilibrium is globally asymptotically stable if it is in the interval of $w$ for which the birth function $b(w)$ is increasing, and that it can remain globally stable if it is larger, but not too much larger, than the value of $w$ at which $b(w)$ attains its maximum, assuming additionally the delay is not too large. 
Acknowledgment. We would like to thank the referees for their very valuable comments.

\section{REFERENCES}

[1] A. Berman and R. J. Plemmons, Nonnegative Matrices in the Mathematical Sciences, Classics Appl. Math. 9, SIAM, Philadelphia, 1994.

[2] G. Bocharov and K. P. Hadeler, Structured population models, conservation laws, and delay equations, J. Differential Equations, 168 (2000), pp. 212-237.

[3] S. A. Gourley AND J. Wu, Extinction and periodic oscillations in an age-structured population model in a patchy environment, J. Math. Anal. Appl., 289 (2004), pp. 431-445.

[4] J. A. J. Metz And O. Diekmann, eds., The Dynamics of Physiologically Structured Populations, Springer-Verlag, New York, 1986.

[5] R. REDLInger, Existence theorems for semilinear parabolic systems with functionals, Nonlinear Anal., 8 (1984), pp. 667-682.

[6] H. L. Smith, Monotone Dynamical Systems. An Introduction to the Theory of Competitive and Cooperative Systems, AMS, Providence, RI, 1995.

[7] H. L. Smith And H. R. Thieme, Strongly order preserving semiflows generated by functionaldifferential equations, J. Differential Equations, 93 (1991), pp. 332-363.

[8] P. Weng, H. Huang, And J. Wu, Asymptotic speed of propagation of wave fronts in a lattice delay differential equation with global interaction, IMA J. Appl. Math., 68 (2003), pp. 409-439.

[9] Y. KuAng, Delay Differential Equations with Applications in Population Dynamics, Academic Press, Boston, MA, 1993. 